\title{
Salivary Alpha-Amylase Activity and Salivary Flow Rate in Young Adults
}

\author{
Aristidis Arhakis ${ }^{1, *}$, Vasilis Karagiannis ${ }^{2}$ and Sotirios Kalfas ${ }^{1}$ \\ ${ }^{1}$ School of Dentistry, Aristotle University of Thessaloniki, Thessaloniki, Greece \\ ${ }^{2}$ School of Mathematics, Aristotle University of Thessaloniki, Thessaloniki, Greece
}

\begin{abstract}
The secretion of salivary alpha-amylase (sAA) is more associated with psychoneuroendocrinological response to stress than with the flow rate and age. The aim of this cross sectional study is to build an explanatory model based on patterns of relationship between age 20-39 in resting and stimulated saliva under no stressful condition in healthy volunteers. Both resting and stimulated saliva were collected from 40 subjects. The sAA values were log-transformed, the normality assumption was verified with the Shapiro-Wilk test and the reliability of the measurements was estimated by the Pearsons' $r$ correlation coefficient. The estimated model was based on the theory of the Linear Mixed Models. Significant mean changes were observed in flow rate and sAA activity between resting and stimulated saliva. The final model consists of two components, the first revealed a positive correlation between age and sAA while the second one revealed a negative correlation between the interaction of age $\times$ flow rate in its condition (resting or stimulated saliva), with sAA. Both flow rate and age influence sAA activity.
\end{abstract}

Keywords: Salivary flow rate, salivary alpha-amylase, age.

\section{INTRODUCTION}

Salivary $\alpha$-Amylase (sAA) is one of the most plentiful components in saliva, accounting for $10-20 \%$ of the total protein content [1]. sAA is locally produced by the highly differentiated epithelial acinar cells of the exocrine salivary glands, mostly of the parotid glands [2]. sAA contributes in food digestion through the hydrolysis of starch to glucose and maltose [3]. Additionaly, sAA has been suggested to prevent bacterial attachment to oral surfaces and to enable bacterial clearance from the mouth [4].

Recent observations indicate a relation between sAA secretion and experience stressful condition. The enzyme concentration increases under both physical stress, such as treadmill exercise, running, bicycle exercise and cold exposure [5-9] and psychological stress as well such as watching highly negative emotional pictures of mutilation or accidents, participating in collegiate level individually oriented athletic competition, written examination, and Trier Social Stress Test (TSST) [10-15]. When the concentration of catecholamines (epinephrine and nor-epinephrine) increases in the blood due to stress, the sAA concentration also increases [16].

Salivary flow rate may be affected from several factors: apart from perceived stress [4] and depression [17] other variables are age [18], alcohol consumption [19], exercise intensity [20], as well as cancer and radiation treatment [21]. In all these studies, the fluctuation of the salivary flow rate coincides with the fluctuation in the sAA concentration.

*Address correspondence to this author at the School of Dentistry, Aristotle University of Thessaloniki, Thessaloniki, Greece; Tel: 00302310253553 ; Fax: 00302310999613; E-mail: oaristidis@gmail.com
However, it is unclear whether the fluctuation of sAA concentration is an effect of the altered salivary flow rate or if it directly depends on the above mentioned stress factors. One study indicates that flow rate is not a confounder of stressinduced sAA activation [22]. Nevertheless, the method used for the determination of the salivary flow rate lacks relevant control. Contrary to other researchers Mackie \& Pangborn (1990) concluded that mastication increases the salivary secretion rate but not the concentration of protein and sAA. They failed to prove a relationship between sAA concentration and salivary flow rate, although they found indications for mastication induced changes in salivary protein concentration [23]. The importance of the sampling techniques, duration times, and locations on sAA determination and the relationship between salivary flow rate and sAA activity, as well, have been pointed out by other authors too [24].

Thus, it remains unclear whether an increase of the salivary flow rate alters the sAA concentration in the absence of a systemically affecting factor. The aim of this cross sectional study was to examine the sAA activity in resting and stimulated saliva sampled from young adults under no stressful condition. An explanatory model is suggested based on patterns of relationship between age, flow rate, and sAA activity in resting and stimulating saliva.

\section{MATERIALS AND METHODOLOGY}

\section{Saliva Samples}

Both resting and stimulated saliva was collected from a total of 40 healthy volunteers, ranged in age from 20 to 39 years (mean age $25.21 \pm 5.06$ years). All subjects were undergraduates, postgraduates, or $\mathrm{PhD}$ dental students and declared medical health. The subjects were informed and 
Table 1. Descriptive Statistics of the Data Set

\begin{tabular}{|l|c|c|c|c|c|c|}
\hline & N & Minimum & Maximum & Median & Mean & Std. Deviation \\
\hline \hline Age & 38 & 20 & 39 & 23.50 & 25.21 & 5.06 \\
\hline flow rate (resting) $\mathrm{ml} / \mathrm{min}$ & 38 & 0.06 & 1.06 & 0.33 & 0.35 & 0.23 \\
\hline flow rate (stimulated) $\mathrm{ml} / \mathrm{min}$ & 38 & 1.00 & 4.10 & 2.18 & 2.22 & 0.74 \\
\hline sAA (resting) U/ml & 38 & 3.09 & 47.08 & 9.91 & 13.01 & 9.28 \\
\hline sAA (stimulated) U/ml & 38 & 5.48 & 56.50 & 17.36 & 20.49 & 11.04 \\
\hline
\end{tabular}

signed a written informed consent. The study protocol was approved by the Ethics committee of the Dental School.

Sampling sessions were limited to the hours between 10:00 and 11:00 AM to minimize the effect of diurnal variations [25]. The subjects were instructed to refrain from exercising [9], eating [26], smoking [27], drinking any beverages [28] except water $1 \mathrm{~h}$ before saliva sampling. Initially, resting whole saliva was collected. The volunteers were supervised and instructed to be comfortably seated with their head tilted slightly forward and to avoid making orofacial movements. Additionally, they were instructed to swallow any saliva in their mouth, immediately before the collection started. In brief, saliva was allowed to accumulate in the floor of the mouth and to drool into the test tube for $15 \mathrm{~min}$ utes [29]. After a 10-minute pause, a consecutive 3-min sample of stimulated saliva was collected. The stimulus was both gustatory and masticatory provided by chewing a flavoured standard sized sugar free gum (ELMA ${ }^{\circledR}$ Chios Greece). The volunteers were asked to chew the gum for 2min and to swallow the saliva produced. Thereafter, they continued chewing the gum for 3 minutes and spit the saliva volume secreted into a test tube. The rate of recurrence of stimulation was approximately 70 chews/min as suggested [29]. The volume of each saliva sample was measured to the tenth of $\mathrm{ml}$. Aliquots of the samples were centrifuged to remove any particulated material and the supernatants were stored at $-80^{\circ} \mathrm{C}$, until analysed [30].

\section{Determination of sAA Activity}

The sAA activity of the samples was detected through the enzymatic hydrolysis of the chromogenic substrate 2-chloro4-nitrophenyl $\alpha$-D maltotrioside [31]. In brief, the reaction mixture contained $5 \mathrm{mM}$ chromogenic substrate, $0.03 \%$ bovine serum albumin, $5 \mathrm{mM} \mathrm{CaCl} 2,50 \mathrm{mM} \mathrm{KSCN}$, and $0.03 \%$ $\mathrm{NaN}_{3}$ in $50 \mathrm{mM}$ Morpholinoethansulfonic acid (MES) buffer, $\mathrm{pH}$ 6.0. The reaction was followed spectrophotometrically at $405 \mathrm{~nm}$.

\section{Statistical Analysis}

From the 40 volunteers following the inclusion criteria 2 were excluded from the analysis since their sAA activity values were $3 \mathrm{SD}$ below the mean value (in at least one of the two conditions stimulated or resting saliva). The values were log-transformed, and the normality assumption was checked with the QQ-plot and Shapiro-Wilk test [32]. The reliability concerning measurements on resting and stimulating values of $\log$ (flow rate) and $\log (\mathrm{sAA})$ were evaluated with Pearson Correlation coefficient $(\mathrm{N}>15)$. Differences between stimulated and resting saliva $\log$ (flow rate) were statistically analyzed with a linear mixed model. Then the $\log$ (flow rate) and $\log (\mathrm{sAA})$ differences between stimulated and resting saliva were computed and the two new variables were afterwards used in two cluster analysis with the kmeans method [33]. Each cluster analysis revealed two cohesive groups of individuals (four groups in overall) and for each one of these groups the correlation between $\log ($ age), differences on $\log$ (flow rate) and differences on $\log (\mathrm{sAA})$ was estimated with Pearson's $r$ correlation coefficient. The results showed some moderate linear relationship between the above mentioned variables. Based on these linear relationships among pairs of groups a series of linear mixed models as conducted with $\log (\mathrm{sAA})-\operatorname{mean}[\log (\mathrm{sAA})]$ as the dependent variable, condition (resting or stimulated saliva flow) as a fixed inter subject factor, subjects as random factor, and $\log ($ age $)-$ mean[log(age) $]$ and $\log ($ flow rate)-mean $[\log ($ flow rate) $]$ as covariates. Each covariate was preferred to be centered at the mean in order to lead to a clearer interpretation of the estimated coefficients [34]. The null hypothesis concerning the significance of the random factor, and the null hypotheses concerning the choice of the bestfitted model were tested with the difference of the -2REML (Restricted Estimated Maximum Likelihood) log-likelihood estimation and Chi-Square test (in the first case the estimation was done with REML while in the second case ML estimation was [34]. The normality assumption for the residuals and the scatter plot of predicted values vs. residuals were used to check the validity of the best fitted model. The analysis was performed with SPSS 16.0 and the level of statistical significance was set at $\mathrm{p}<0.05$.

\section{Results}

Descriptive statistics of the data set are shown in Table $\mathbf{1 .}$ A statistically significant increase in flow rate was observed, between resting and stimulated conditions $(p<0.001$, mixed linear model with condition as repeated effect and subject as a random factor). The reliability coefficients concerning measurements on resting and stimulated values of $\log$ (flow rate) and $\log (\mathrm{sAA})$ were $r=0.51$ and $r=0.6(p<0.001$, Pearson's correlation coefficient), respectively. Both coefficients are less than 0.7 [in order to compare groups, the intraclass correlation coefficient must be greater or equal to 0.7] [35] this proving the existence of intersubject heterogeneity. As suggested $[32,33]$ the delta scores (stimulated minus resting values) were calculated for $\log$ (flow rate) and $\log (\mathrm{sAA})$ activity and the resulted variables were denoted by Dlog(flow rate) and $\mathrm{D} \log (\mathrm{sAA})$, correspondingly. Dlog(flow rate) and D $\log (\mathrm{sAA})$ were used subsequently in two cluster analyses with the $k$-means method [33]. Fig. (1) and Fig. (2) depict the scatterplots of Dlog(flow rate) vs. Dlog(sAA) according to the results of the two cluster analyses. Considering the 


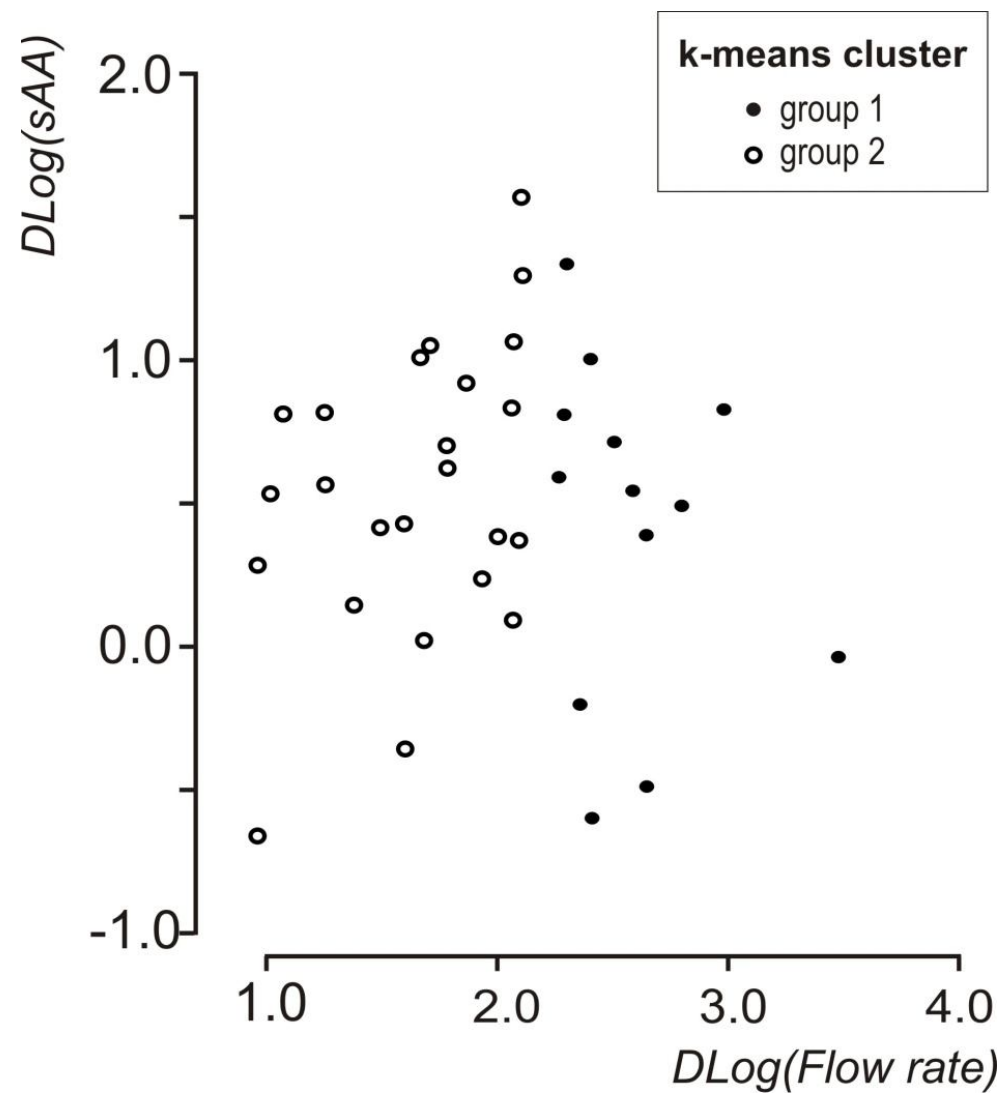

Fig. (1). Scatterplot of Dlog(flow rate) vs Dlog(sAA) showing the groups that emerged after the k-means cluster analysis on Dlog(flow rate).

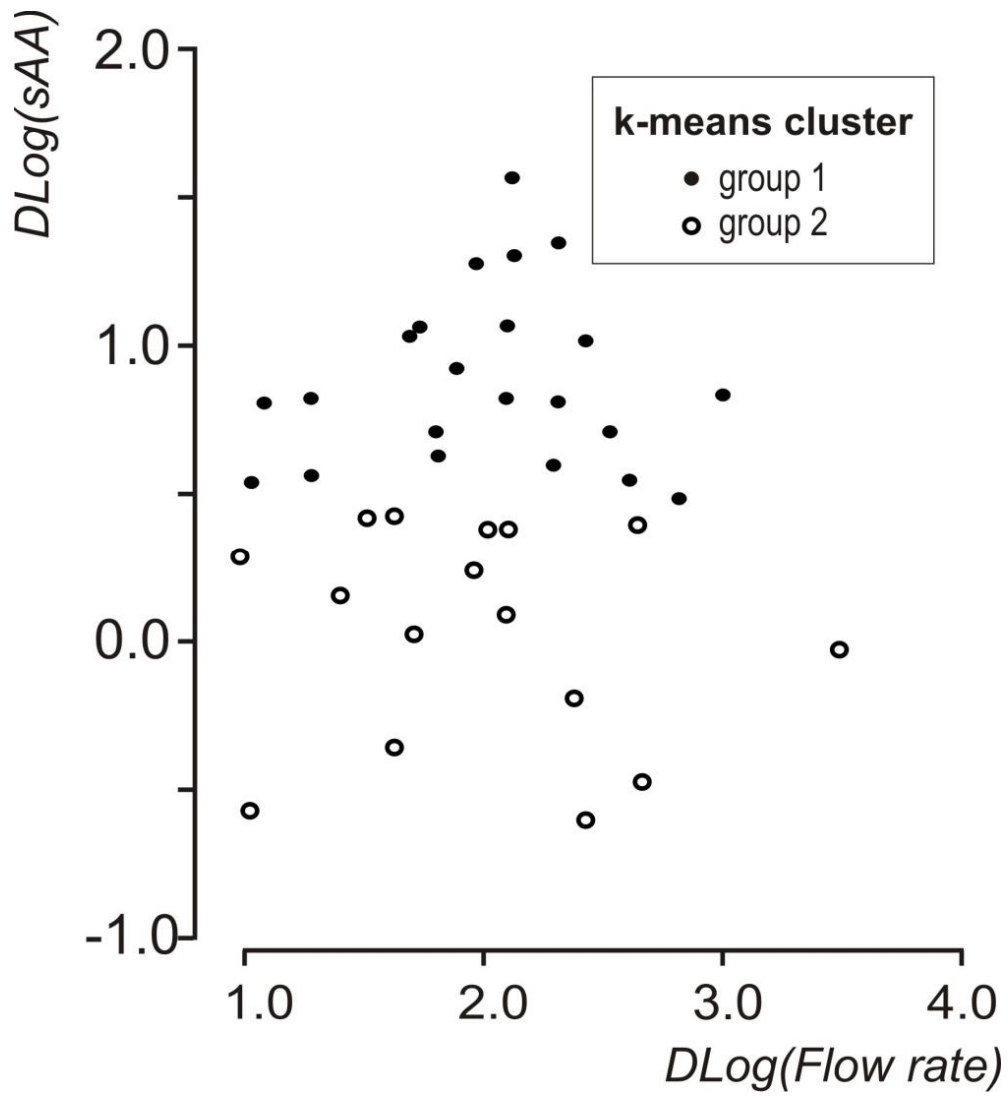

Fig. (2). Scatterplot of Dlog(flow rate) vs Dlog(sAA) showing the groups that emerged after the k-means cluster analysis on Dlog(sAA). 


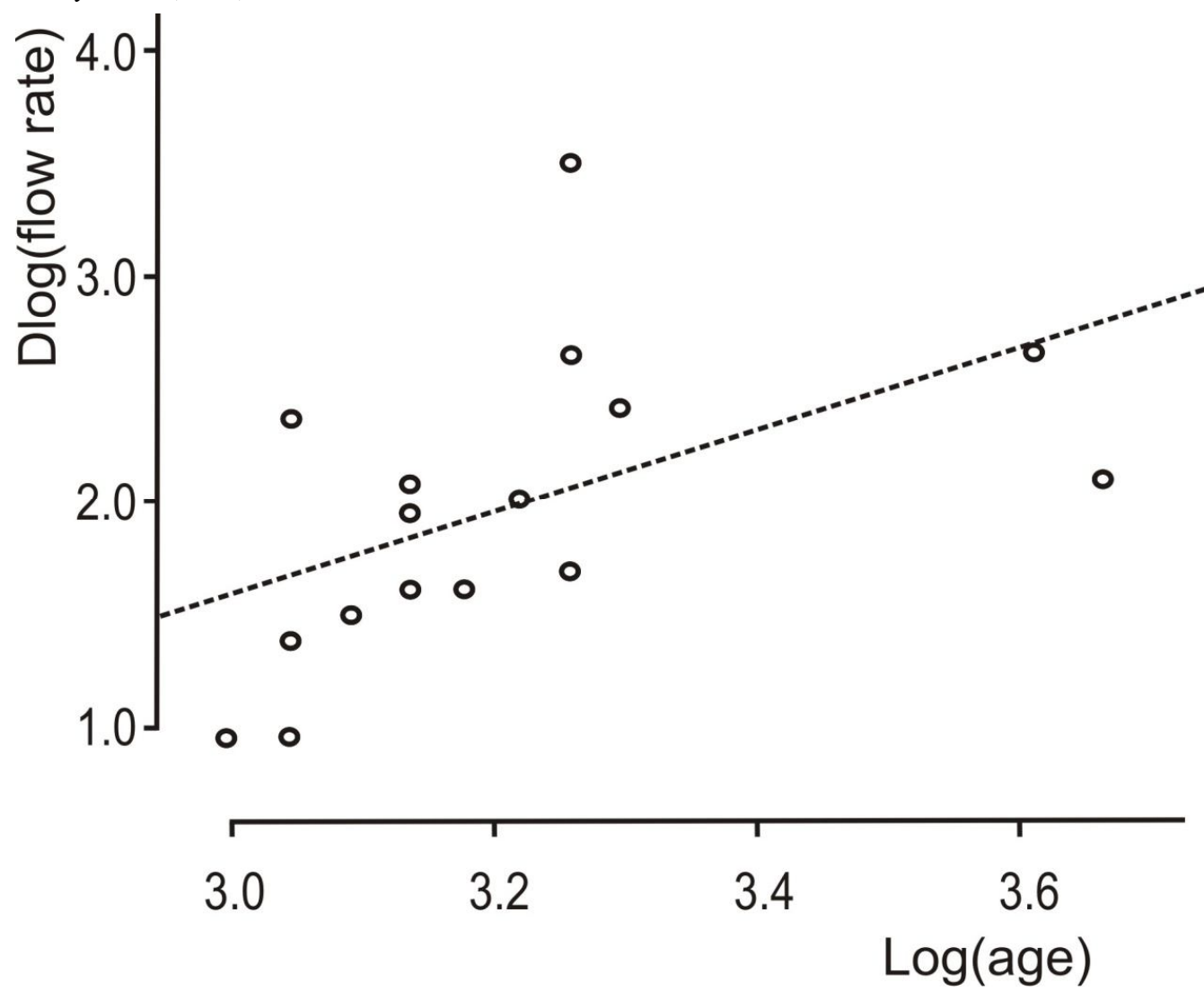

Fig. (3). Scatterplot of $\log ($ age $)$ vs. D $\log$ (flow rate), showing subjects with decreased $\operatorname{Dlog}(\mathrm{sAA}), r=0.51, \mathrm{~N}=16$ (Dlog(sAA) $<0.5)$.

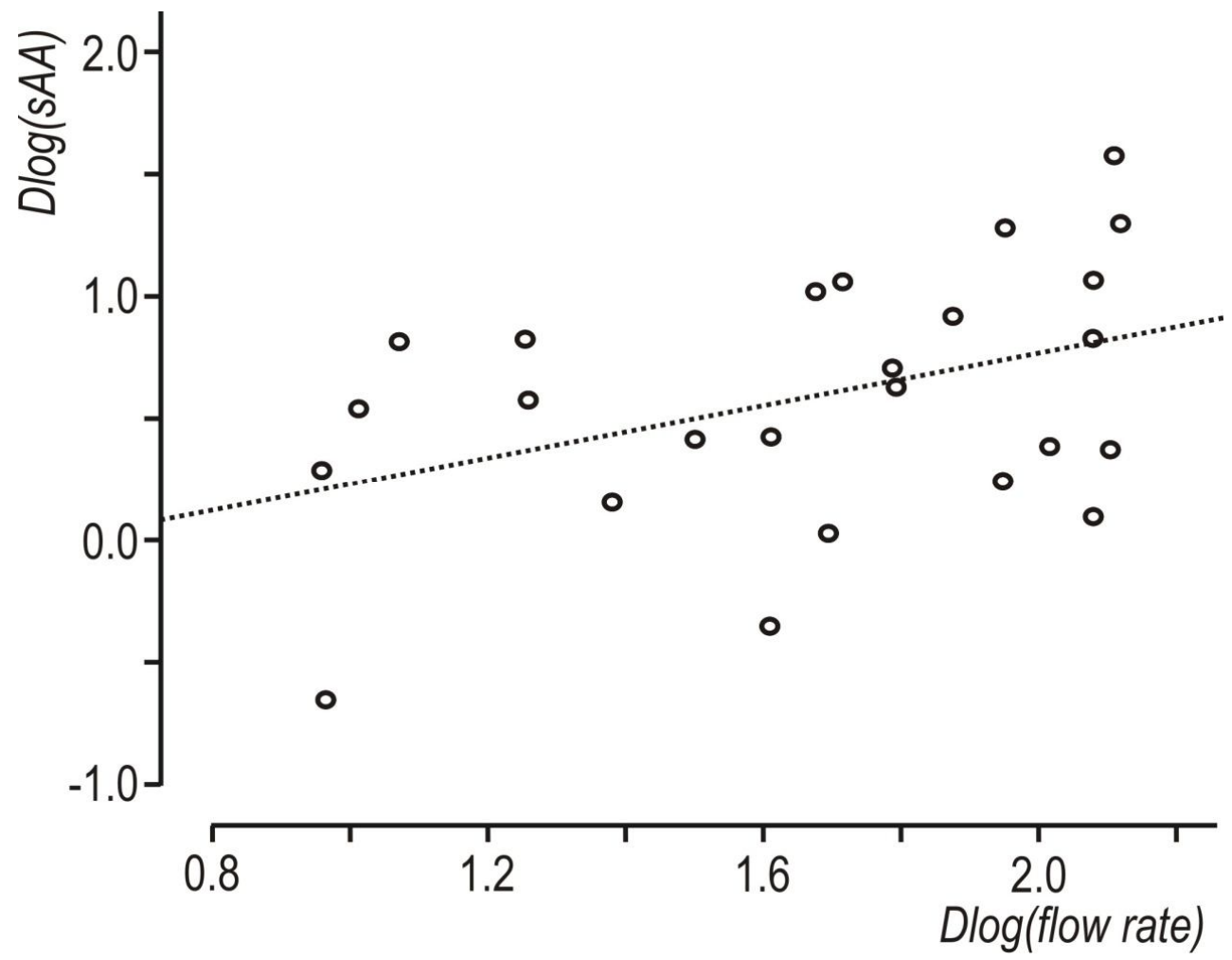

Fig. (4). Scatterplot of D $\log$ (flow rate) vs D $\log (\mathrm{sAA})$, showing subjects with decreased $\operatorname{Dlog}($ flow rate), $r=0.41 \mathrm{~N}=25$ (Dlog(flow rate) $<2.1$ ).

groups that emerged after the cluster analyses some moderate correlations were found between Dlog(flow rate), D $\log (\mathrm{sAA})$ and $\log ($ age $)$. For subjects with decreased $\mathrm{D} \log (\mathrm{sAA})<0.5$, the correlation between $\log ($ age $)$ and
Dlog(flow rate) was $r=0.51, p=0.042(\mathrm{~N}=16)$ (Fig. 3), whereas for subjects with decreased $D \log ($ flow rate $)<2.1$, the correlation between $\operatorname{Dlog}$ (flow rate) and $\mathrm{D} \log (\mathrm{sAA})$ was $r=0.41, p=0.042(\mathrm{~N}=25)$ (Fig. 4). Based on these findings, a 
Table 2. Results Concerning Hypothesis About Fixed Effects in the Full Linear Mixed Model, F(Numerator df, Denominator df) is the Corresponding Value of the F Distribution, with Corrected Degrees of Freedom

\begin{tabular}{|c|c|c|c|c|}
\hline Source & Numerator df & Denominator df & $\mathbf{F}$ & $p$-Value \\
\hline Intercept & 1 & 36.580 & 1347.714 & $<0.001$ \\
\hline $\log ($ age $)-$ mean $[\log ($ age $)]$ & 1 & 38.860 & 14.637 & $<0.001$ \\
\hline Condition & 1 & 36.586 & 45.140 & $<0.001$ \\
\hline $\log ($ flow rate $)-\operatorname{mean}[\log ($ flow rate $)$ & 1 & 57.711 & .000 & .984 \\
\hline$\{\log ($ flow rate $)-$ mean $[\log ($ flow rate $)\} \times \log ($ age $)-\operatorname{mean}[\log ($ age $)]$ & 1 & 58.698 & 11.334 & 0.001 \\
\hline condition $\times\{\log ($ flow rate $)-$ mean $[\log ($ flow rate $)\}$ & 1 & 35.659 & .540 & .467 \\
\hline condition $\times\{\log ($ age $)-\operatorname{mean}[\log ($ age $)]\}$ & 1 & 39.575 & .014 & .908 \\
\hline condition $\times\{\log ($ flow rate $)-$ mean $[\log ($ flow rate $)\} \times \log ($ age $)-$ mean $[\log ($ age $)]$ & 1 & 35.844 & .997 & .325 \\
\hline
\end{tabular}

Table 3. Results Concerning Hypothesis about Fixed Effects in the Restricted Linear Mixed Model, F(numerator df, Denominator df) is the Corresponding Value of the F Distribution, with Corrected Degrees of Freedom

\begin{tabular}{|c|c|c|c|c|}
\hline Source & Numerator df & Denominator df & $\mathbf{F}$ & p-value \\
\hline Intercept & 1 & 38.642 & 1539.503 & $<0.001$ \\
\hline $\log (\operatorname{age})-\operatorname{mean}[\log ($ age $)]$ & 1 & 38.962 & 17.753 & $<0.001$ \\
\hline condition & 1 & 38.532 & 48.468 & $<0.001$ \\
\hline$\{\log ($ flow rate $)-\operatorname{mean}[\log ($ flow rate $)\} \times \log ($ age $)-$ mean $[\log ($ age $)]$ & 1 & 55.228 & 12.899 & 0.001 \\
\hline
\end{tabular}

Table 4. Fixed Effects Parameter Estimates in the Restricted Model

\begin{tabular}{|c|c|c|c|c|c|c|c|c|}
\hline \multirow{2}{*}{\multicolumn{2}{|c|}{ Parameter }} & \multirow{2}{*}{ Estimate } & \multirow{2}{*}{ Std. Error } & \multirow{2}{*}{ df } & \multirow{2}{*}{$\mathbf{t}$} & \multirow{2}{*}{$p$-Value } & \multicolumn{2}{|c|}{ 95\% Confidence Interval } \\
\hline & & & & & & & Lower Bound & Upper Bound \\
\hline \multicolumn{2}{|c|}{ Intercept } & 2.942 & 0.074 & 40.592 & 39.997 & $<0.001$ & 2.794 & 3.091 \\
\hline \multicolumn{2}{|c|}{ b: $\log ($ age $)-\operatorname{mean}[\log ($ age $)]$} & 1.560 & 0.370 & 38.962 & 4.213 & $<0.001$ & 0.811 & 2.310 \\
\hline \multirow{2}{*}{ c: Condition } & resting & -0.572 & 0.082 & 38.532 & -6.962 & $<0.001$ & -0.738 & -0.406 \\
\hline & stimulated & $0^{\mathrm{a}}$ & 0 & . & . & . & . & . \\
\hline \multicolumn{2}{|c|}{$\begin{array}{l}\text { fa: }\{\log (\text { flow rate })-\text { mean }[\log (\text { flow } \\
\text { rate })\} \times\{\log (\text { age })-\text { mean }[\log (\text { age })]\}\end{array}$} & -2.253 & 0.627 & 55.228 & -3.592 & 0.001 & -3.510 & -0.996 \\
\hline
\end{tabular}

${ }^{\mathrm{a}}$ :This parameter is set to zero because it is redundant.

Functional form of the proposed model sAA activity $=\mathrm{e}^{2.942+1.56 \times \mathrm{b}-0.572 \times \mathrm{c}-2.253 \times \mathrm{fa}}$

linear mixed model was fitted to the data set, with the $\log (\mathrm{sAA})$ as the dependent variable, the condition (resting or stimulated) as the fixed within factor, the subject as random factor and the covariates $\log ($ age $)$-mean[log(age) $]$ and $\log$ (flow rate) - mean[log(flow rate)] centered at the mean [34]. Results concerning the effect of the fixed factor the covariates and all their interactions (called fixed effects) under the full model are presented in Table 2. The likelihood ratio statistic of the significance of the random factor was calculated by subtracting the value of the -2REML loglikelihood of the full model without the random factor from the corresponding value of the full model containing the random factor, i.e. $-99.391-(-108.274)=8.883$. The $p$-value for the likelihood ratio statistic, was $P\left(\mathrm{X}^{2}(1)>8.883\right)=0.005$, thus, revealing that the effect of the random factor (subject) is statistically significant and must be kept in the analysis. After the exclusion of the non-significant parameters from the full model (Table 2 , see any line with $p>0.05$ ) a new linear mixed model was conducted (restricted model) and the results about the remaining fixed effects and their parameters are shown in Table 3 and Table $\mathbf{4}$, respectively. The likelihood ratio statistic for the comparison between the full and the restricted model was calculated by subtracting the value of the $-2 \mathrm{ML}$ log-likelihood for the restricted model from the corresponding value of the full model, i.e. -92.244 - ($94.508)=2.264$. The $p$-value for the likelihood ratio statistic was $P\left(X^{2}(4)>2.264\right)=0.687$ indicating that the restricted model is better than the full model. The conditional residuals of the restricted model appear to follow a normal distribution [Kolmogorov-Smirnov test, $p=0.200$ and QQ-plot, (Fig. 5)]. The assumption of the constant variance for the residuals in the restricted model can be verified from the scatterplot of conditional predicted values vs. conditional residuals (Fig. 6). The chart shows no particular pattern and the values of the residuals seem to be symmetric around zero).

The results from the restricted model revealed:

(a) a statistically significant increase in $\log (\mathrm{sAA})$ at the stimulating condition $(p<0.001$, Table 4 , coefficient for condition $=$ rest $)$, 


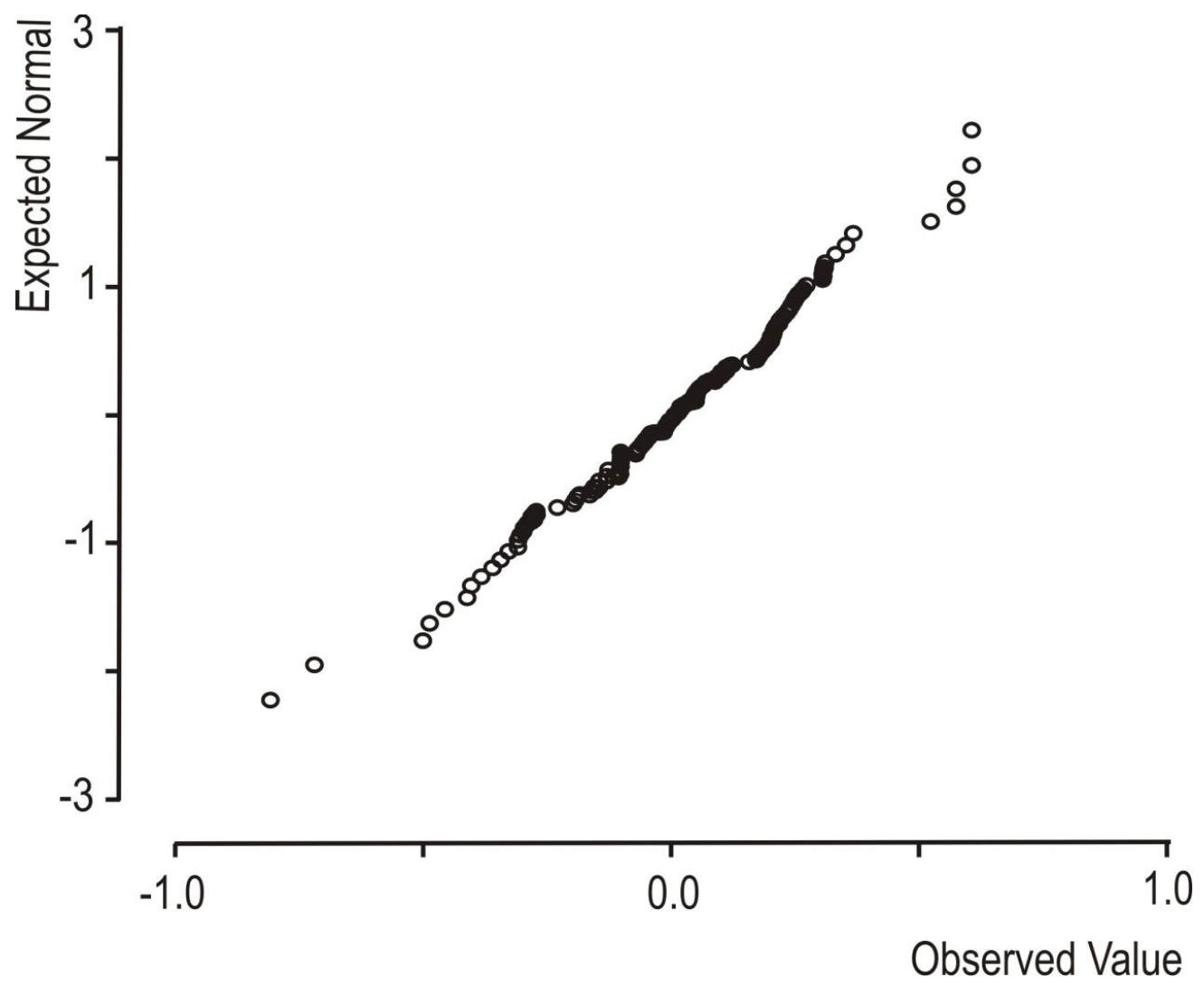

Fig. (5). Normal QQ-plot of the conditional residuals from the restricted (best) model.

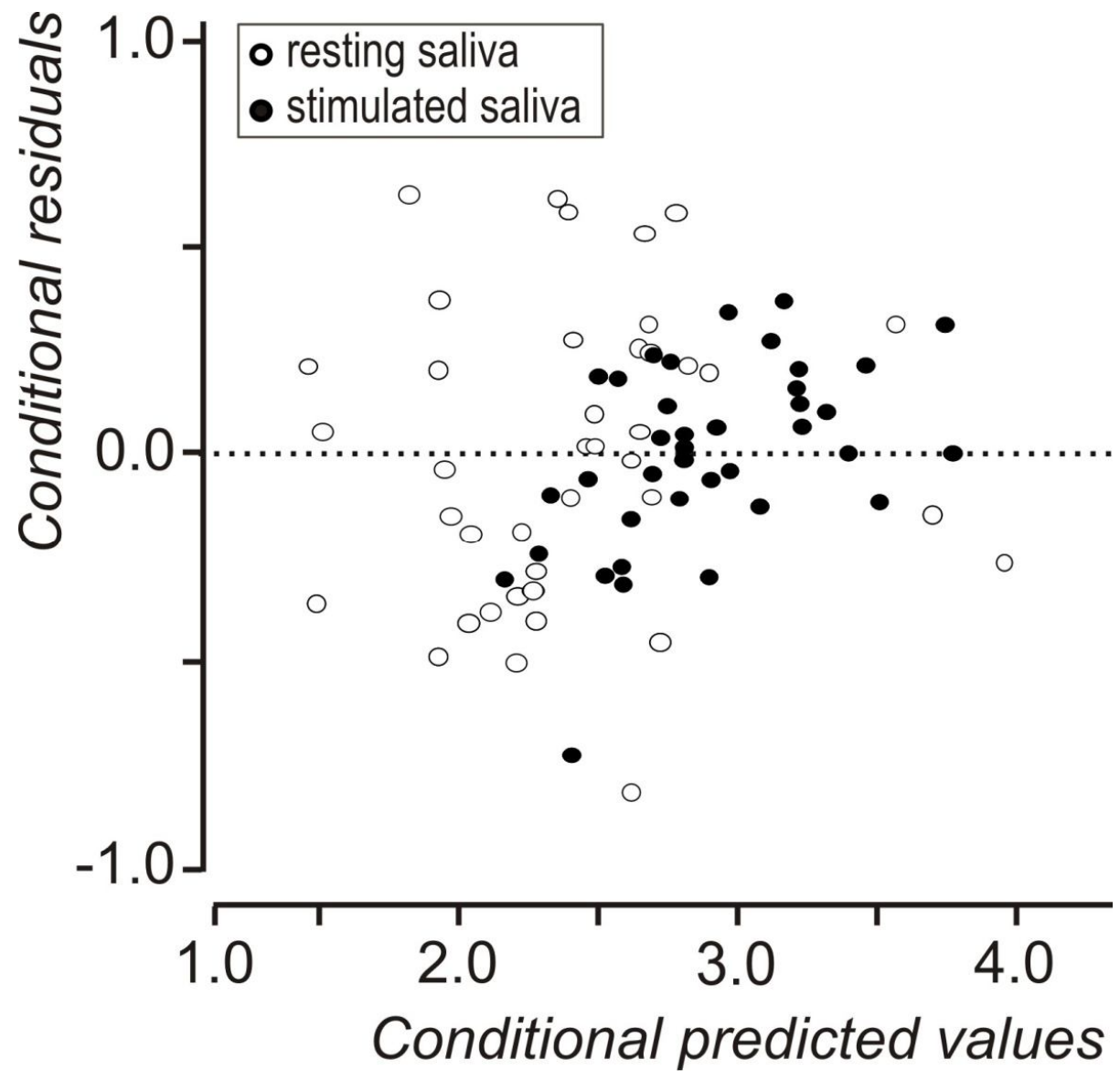

Fig. (6). Conditional predicted values vs conditional residuals of the restricted (best) model. 
(b) a positive association between sAA and age $(p<0.001$, Table 4, coefficient for $\{\log ($ age $)-$ mean $[\log ($ age $)]\})$ and

(c) a negative association between sAA and the interaction of flow rate and age $(p=0.001$, Table 4 , coefficient for $\{\log ($ flow rate $)-$ mean $[\log ($ flow rate $)\} \times\{\log ($ age $)-$ mean $[\log ($ age $)]\})$.

\section{DISCUSSION}

As a surrogate non-invasive indicator of Sympathetic Nervous System (SNS) activation, sAA has widely been assessed the last two decades. An important concern is whether the salivary flow rate is a confounding factor that modifies sAA concentration. Saliva is a complex mixture derived from many different cell types within the glands innervated by several nerve types. Sympathetic stimulation decreases the salivary flow rate and increases the total protein salivary content [36] Increase of the salivary flow rate is due to parasympathetic nervous pathways stimulation, which is mainly responsible for regulating salivary flow rate [37] Our study was conducted under no stressful conditions, meaning that the parasympathetic stimulation mainly affected the flow rate and to a lesser extent the sAA secretion. The results revealed a 1.5 times mean increase of sAA in the stimulated saliva compared with the resting saliva, while the flow rate increased 6.3 times. Other studies measuring sympathetic stimulation (i.e. stressful conditions such as academic examination, skydiving, playing a stressful video game, watching a gruesome video, Trier Social Stress Test) showed sAA increase varied from 1.4-2.5 [4, 8, 22, 38-40]. Thus, the proportional increase of the sAA due to saliva flow increase under no stressful condition is of similar magnitude with the one recorded under stress.

sAA activity as a valid and reliable measure of SNS activity is not definite, as long as the influence of PNS on the sAA is not negligible. sAA concentrations can be influenced via synergistic sympathetic-parasympathetic interactions whereby parasympathetic activity amplifies sympathetic effects, and via parasympathetic activity through glands that are solely or mainly parasympathetically innervated, or through the effects of salivary flow rate (parasympathetically-mediated) [41]. Our results are in line with that showing the salivary flow rate as a confounder in sAA measurement. On the contrary Rohleder et al (2006) concluded that saliva flow rate have a minimal impact on sAA output [22]. This conclusion was mainly derived from experiments with small effects on flow rate like the one of the collection method. Similar findings were reported by others [38]. However, changes in salivary fluid secretion account for $25-40 \%$ changes in sAA activity [42], this implying the important role of salivary flow rate. A very recent study is in line with our findings strongly disputing the role of sAA as a marker of the SNS and suggesting that salivary chromogranin A but not sAA, correlates with cardiovascular parameters during high intensity exercise, confirming salivary chromogranin A as marker of psychological stress related to physical activity.

Based on the present findings a statistical explanatory model is suggested that defines the association between salivary flow rate, age, and sAA. The increase of sAA follows the increase of salivary flow rate under no stressful condition.
This finding implies a triggering on the acinar cells of the salivary glands for higher sAA production. This is in line with a study searching the effect of oral stimulation on human parotid salivary flow rate and sAA secretion [43]. It is not well known whether the human parotid gland contains a sufficient store of sAA for continual secretion or whether sAA synthesis is stimulated and sufficiently rapid to replace the sAA being secreted. It was shown, though, that the parotid gland was able to maintain a high protein output for a long period of time [44]. Additionally resting saliva is mainly secreted by the submandibular glands and only about $20 \%$ derives from the parotid gland, which happens to be very rich in sAA $[45,46]$. Masticatory and gustatory stimulation drastically changes salivary protein composition due to the different responsivity of the parotid and submandibular glands to stimulation and the different amounts of sAA and other proteins that the secretion of these glands contains. Thus, in stimulated saliva the contribution of individual glands changes, whereby about half of all saliva is from the parotid glands. This condition highly affects sAA concentration in whole saliva as parotid saliva contains a 4-10-fold higher sAA amount than submandibular saliva [47]. Based on that it is recommended to specify the salivary flow rate for the sAA activity determination. Most of the studies measuring sympathetic stimulation through sAA activation [4, $8,22,38-40]$ disregard this parameter. Rohleder et al 2006 collected stimulated and unstimulated saliva under stressful condition (TSST). There was 2.7 times sAA increase in resting saliva and 1.8 in stimulated saliva. The synergistic effect of sympathetic, due to stress, and parasympathetic system, due to mastication on stimulated saliva reduced the sAA levels [22].

The statistical analysis of the present results revealed an influence of age on sAA activity. The relationship between flow rate and age changes through life span; in newborns the sAA activity is detected very low [48-50] and climbs to the adult levels within the first 3 years $[51,52]$. Over the adulthood until older ages the sAA activity seems to remain unchanged [53,54]. Studies have shown that with increasing age morphological changes of oral mucosa appear but only marginal alterations of salivary gland function and saliva composition are seen [55]. Nagler and Hershkovich (2005) reported a $62 \%$ lower resting saliva flow rate and higher sAA concentrations in elderly participants compared to young adults [56]. All the studies related with the sAA activity fluctuations in adulthood, categorised the subjects in age groups and proceed with a comparison between these age groups. In our study we focused in the subjects of one age group only and found a positive association between age and sAA activity in young adults 20 to 39 years old. Finally a negative association of the sAA is observed with the interaction between flow rate and age.

To conclude, in the absence of a stressful stimulus, the flow rate, age as well as the interaction of these two factors affect the secretion of sAA in healthy young adults.

\section{CONFLICT OF INTEREST}

The authors confirm that this article content has no conflicts of interest.

\section{ACKNOWLEDGEMENTS}

Declared none. 


\section{REFERENCES}

[1] Baum BJ. Principles of saliva secretion. Ann N Y Acad Sci 1993; 694: 17-23.

[2] Castle D, Castle A. Intracellular transport and secretion of salivary proteins. Crit Rev Oral Biol Med 1998; 9: 4-22.

[3] Zakowski JJ, Bruns DE. Biochemistry of human alpha amylase isoenzymes. Crit Rev Clin Lab Sci 1985; 21: 283-322.

[4] Bosch JA, De Geus EJ, Veerman EC, Hoogstraten J, Nieuw Amerongen AV. Innate secretory immunity in response to laboratory stressors that evoke distinct patterns of cardiac autonomic activity. Psychosom Med 2003; 65: 245-58.

[5] Gilman S, Thornton R, Miller D, Biersner R. Effects of exercise stress on parotid gland secretion. Horm Metab Res 1979; 11: 454.

[6] Nexø E, Hansen MR, Konradsen L. Human salivary epidermal growth factor, haptocorrin and amylase before and after prolonged exercise. Scand J Clin Lab Invest 1988; 48: 269-73.

[7] Steerenberg PA, Van Asperen IA, van Nieuw Amerongen A, et al. Salivary levels of immunoglobulin A in triathletes. Eur J Oral Sci 1997; 105: 305-9.

[8] Chatterton RT Jr., Vogelsong KM, Lu YC, Hudgens GA. Hormonal responses to psychological stress in men preparing for skydiving. J Clin Endocrinol Metab 1997; 82: 2503-9.

[9] Walsh NP, Blannin AK, Clark AM, et al. The effects of highintensity intermittent exercise on saliva IgA, total protein and alpha-amylase. J Sports Sci 1999; 17: 129-34.

[10] Van Stegeren A, Rohleder N, Everaerd W, Wolf OT. Salivary alpha amylase as marker for adrenergic activity during stress: effect of betablockade. Psychoneuroendocrinology 2006; 31: 137-41.

[11] Kivlighan KT, Granger DA. Salivary alpha-amylase response to competition: relation to gender, previous experience, and attitudes. Psychoneuroendocrinology 2006; 31: 703-14.

[12] Rohleder N, Nater UM, Wolf JM, Ehlert U, Kirschbaum C. Psychosocial stress-induced activation of salivary alpha-amylase: an indicator of sympathetic activity? Ann N Y Acad Sci 2004; 1032: 258-63.

[13] Gordis EB, Granger DA, Susman EJ, Trickett PK. Asymmetry between salivary cortisol and alpha-amylase reactivity to stress: relation to aggressive behavior in adolescents. Psychoneuroendocrinology 2006; 31: 976-87.

[14] Nater UM, Rohleder N, Gaab J, et al. Human salivary alphaamylase reactivity in a psychosocial stress paradigm. Int $\mathrm{J}$ Psychophysiol 2005; 55: 333-42.

[15] Nater UM, La Marca R, Florin L, et al. Stress-induced changes in human salivary alpha-amylase activity-associations with adrenergic activity. Psychoneuroendocrinology 2006; 31: 49-58.

[16] Chatterton RTJR, Vogelsong KM, Lu YC, Ellman AB, Hudgens GA. Salivary alpha-amylase as a measure of endogenous adrenergic activity. Clin Physiol 1996; 16: 433-48.

[17] Bergdahl M, Bergdahl J. Perceived taste disturbance in adults: prevalence and association with oral and psychological factors and medication. Clin Oral Investig 2002; 6: 145-9.

[18] Dezan CC, Nicolau J, Souza DN, Walter LR. Flow rate, amylase activity, and protein and sialic acid concentrations of saliva from children aged 18, 30 and 42 months attending a baby clinic. Arch Oral Biol 2002; 47: 423-7.

[19] Enberg N, Alho H, Loimaranta V, Lenander-Lumikari M. Saliva flow rate, amylase activity, and protein and electrolyte concentrations in saliva after acute alcohol consumption. Oral Surg Oral Med Oral Pathol Oral Radiol Endod 2001; 92: 292-8.

[20] Allgrove JE, Gomes E, Hough J, Gleeson M. Effects of exercise intensity on salivary antimicrobial proteins and markers of stress in active men. J Sports Sci 2008; 26: 653-61.

[21] Chitra S, Shyamala Devi CS. Effects of radiation and alphatocopherol on saliva flow rate, amylase activity, total protein and electrolyte levels in oral cavity cancer. Indian J Dent Res 2008; 19: 213-8.

[22] Rohleder N, Wolf JM, Maldonado EF, Kirschbaum C. The psychosocial stress -induced increase in salivary alpha-amylase is independent of saliva flow rate. Psychophysiology 2006; 43: 645-52.

[23] Mackie DA, Pangborn RM. Mastication and its influence on human salivary flow and alpha-amylase secretion. Physiol Behav 1990; 47: 593-5.

[24] Beltzer EK, Fortunato CK, Guaderrama MM, et al. Salivary flow and alpha-amylase: collection technique, duration, and oral fluid type. Physiol Behav 2010; 101: 289-96.
[25] Nater UM, Rohleder N, Schlotz W, Ehlert U, Kirschbaum C. Determinants of the diurnal course of salivary alpha-amylase. Psychoneuroendocrinology 2007; 32: 392-401.

[26] Messenger B, Clifford MN, Morgan LM. Glucose-dependent insulinotropic polypeptide and insulin-like immunoreactivity in saliva following sham-fed and swallowed meals. J Endocrinol 2003; 177: 407-12.

[27] Zappacosta B, Persichilli S, Mordente A, et al. Inhibition of salivary enzymes by cigarette smoke and the protective role of glutathione. Hum Exp Toxicol 2002; 21: 7-11.

[28] Brand HS, Bruins ML, Veerman EC, Nieuw Amerongen AV. Secretion rate and amylase concentration of whole saliva after consumption of beer. Int J Dent Hyg 2006; 4: 160-1.

[29] Navazesh M. Methods for collecting saliva. Ann NY Acad Sci 1993; 694: 72-7.

[30] Granger DA, Kivlighan KT, El-Sheikh M, Gordis EB, Stroud LR. Salivary alpha-amylase in biobehavioral research: recent developments and applications. Ann NY Acad Sci 2007; 1098: 122-44.

[31] Winn-Deen ES, David H, Sigler G, Chavez R. Development of a direct assay for alpha-amylase. Clin Chem 1988; 34: 2005-8.

[32] Rohleder N, Nater UM. Determinants of salivary alpha-amylase in humans and methodological considerations. Psychoneuroendocrinology 2009; 34: 469-85.

[33] Pruessner JC, Kirschbaum C, Meinlschmid G, Hellhammer DH. Two formulas for computation of the area under the curve represent measures of total hormone concentration versus time-dependent change. Psychoneuroendocrinology 2003; 28: 916-31.

[34] West B, Welch K, Galecki A. Linear mixed models. a practical guide using statistical software. Boca Raton: Chapman \& Hall/CRC 2007.

[35] Scientific Advisory Committee of the Medical Outcomes Trust Source. Assessing health status and quality-of-life instruments: attributes and review criteria. Qual Life Res 2002; 11: 193-205.

[36] Proctor GB, Carpenter GH. Regulation of salivary gland function by autonomic nerves. Auton Neurosci 2007; 133: 3-18.

[37] Garrett JR. The proper role of nerves in salivary secretion: a review. J Dent Res 1987; 66: 387-97.

[38] Bosch JA, Brand HS, Ligtenberg TJ, et al. Psychological stress as a determinant of protein levels and salivary-induced aggregation of Streptococcus gordonii in human whole saliva. Psychosom Med 1996; 58: 374-82.

[39] Skosnik PD, Chatterton RT JR, Swisher T, Park S. Modulation of attentional inhibition by norepinephrine and cortisol after psychological stress. Int J Psychophysiol 2000; 36: 59-68.

[40] Takai N, Yamaguchi M, Aragaki T, et al. Effect of psychological stress on the salivary cortisol and amylase levels in healthy young adults. Arch Oral Biol 2004; 49: 963-8.

[41] Bosch JA, Veerman EC, De Geus EJ, Proctor GB. $\alpha$-Amylase as a reliable and convenient measure of sympathetic activity: don't start salivating just yet! Psychoneuroendocrinology 2011; 36: 449-53.

[42] Gallina S, Di Mauro M, D'amico MA, et al. Salivary chromogranin A, but not $\alpha$-amylase, correlates with cardiovascular parameters during high-intensity exercise. Clin Endocrinol 2011; 75: 747-52.

[43] Froehlich DA, Pangborn RM, Whitaker JR. The effect of oral stimulation on human parotid salivary flow rate and alpha-amylase secretion. Physiol Behav 1987; 41: 209-17.

[44] Dawes C. The composition f human saliva secreted in response to a gustatory stimulus and to pilocaprine. J Physiol 1966; 183: 360-8.

[45] Schenkels LC, Veerman EC, Nieuw Amerongen AV. Biochemical composition of human saliva in relation to other mucosal fluids. Crit Rev Oral Biol Med 1995; 6: 161-75.

[46] Humphrey SP, Williamson RT. A review of saliva: normal composition, flow, and function. J Prosthet Dent 2001; 85: 162-9.

[47] Veerman EC, Van Den Keybus PA, Vissink A, Nieuw Amerongen AV. Human glandular salivas: their separate collection and analysis. Eur J Oral Sci 1996; 104: 346-52.

[48] Bellavia SL, Moreno J, Sanz E, Picas EI, Blanco A. alpha-Amylase activity of human neonate and adult saliva. Arch Oral Biol 1979; 24: 117-21

[49] Hodge C, Lebenthal E, Lee PC, Topper W. Amylase in the saliva and in the gastric aspirates of premature infants: its potential role in glucose polymer hydrolysis. Pediatr Res 1983; 17: 998-1001.

[50] Sevenhuysen GP, Holodinsky C, Dawes C. Development of salivary alpha-amylase in infants from birth to 5 months. Am J Clin Nutr 1984; 39: 584-98. 
[51] Davis EP, Granger DA. Developmental differences in infant salivary alpha-amylase and cortisol responses to stress. Psychoneuroendocrinology 2009; 34: 795-804.

[52] Yim IS, Granger DA, Quas JA. Children's and adults' salivary alpha-amylase responses to a laboratory stressor and to verbal recall of the stressor. Dev Psychobiol 2010; 52: 598-602.

[53] Ben-Aryeh H, Shalev A, Szargel R, et al. The salivary flow rate and composition of whole and parotid resting and stimulated saliva in young and old healthy subjects. Biochem Med Metab Biol 1986; 36: $260-5$.
[54] Aguirre A, Levine MJ, Cohen RE, Tabak LA. Immunochemical quantitation of alpha-amylase and secretory $\operatorname{IgA}$ in parotid saliva from people of various ages. Arch Oral Biol 1987; 32: 297-301.

[55] Ghezzi EM, Ship JA. Aging and secretory reserve capacity of major salivary glands. J Dent Res 2003; 82: 844-8.

[56] Nagler RM, Hershkovich O. Relationships between age, drugs, oral sensorial complaints and salivary profile. Arch Oral Biol 2005; 50: 7-16.

Received: September 25, 2012

(C) Arhakis et al.; Licensee Bentham Open.

This is an open access article licensed under the terms of the Creative Commons Attribution Non-Commercial License (http://creativecommons.org/licenses/by-nc/3.0/) which permits unrestricted, non-commercial use, distribution and reproduction in any medium, provided the work is properly cited. 